\title{
FHQA JDRA Schemes for Reliability and Energy Efficiency - Soft-DF-based OR-Assisted SC-FDMA
}

\author{
D.Prabakar \\ Assistant Professor, ECE \\ Prist University \\ Puducherry Campus
}

\author{
C.Gowthami \\ M.Tech Student, ECE \\ Prist University \\ Puducherry Campus
}

\begin{abstract}
In this paper, we exploit the benefits of the diversity gains that arise from a cluster of opportunistic relays (ORs) and from the independently fading subcarriers of multiple users. Our goal is to improve the energy efficiency of the decodeandforward-based OR-assisted single-carrier frequency-division multiple-access (SC-FDMA) uplink, where the direct transmission (DT) link is unavailable. We proposed two joint dynamic resource allocation (JDRA) schemes by assuming that the pilotaided channel-quality information (CQI) of all the users may be exchanged. Furthermore, we take the following two main aspects into account: 1) "first-hop quality awareness" (FHQA) for JDRA-aided OR and 2) "buffering delay awareness" in the context of interleaver-aided channelcoded systems. In addition, frequency-domain turbo equalizers are employed in both the relay and BS's receivers. Our results demonstrate that, compared to the DT benchmarker, the proposed FHQA JDRA schemes can achieve an energy reduction gain of $91 \%$ for a single-antenna base station (BS) receiver and up to $7.4 \%$ for a multiantenna BS when considering the energy consumption due to CQI exchange among relays.
\end{abstract}

\section{Keywords}

Orthogonal frequency division multiplexing (OFDM), peakto-average power ratio (PAPR), partial transmit sequence, selected mapping.

\section{INTRODUCTION}

Relay-assisted wireless communications have been explored for diverse ad hoc and cellular networks to improve the attainable spectral or energy efficiency of classic direct transmissions (DTs) [1]. Naturally, the availability of inactive mobiles as candidate relays has the potential tomitigate the effect of fading. The activation of multiple relays results in cooperative diversity. When dynamically reassigning the relays based on their location or channel quality, we arrive at the concept of opportunistic relaying (OR) [2].

Furthermore, orthogonal frequency-division multiplexing (OFDM)-style broadband frequency-division multiple-access (FDMA) systems, such as the Third-Generation Partnership Project Long Term Evolution (3GPP LTE) system's uplink single-carrier frequency-division multiple-access (SC-FDMA) scheme [3] and orthogonal frequency-division multiple-access (OFDMA) [4], conveniently facilitate near-instantaneous adaptive subband/subcarrier allocation and multiuser (MU) scheduling. This is achieved by exploiting the knowledge of the time-varying channel-state information (CSI) of the subbands/ subcarriers [5]-[9] when communicating over frequencyselective fading channels.
Clearly, directly adopting the conventional OR concept in [2] to OFDM-based systems at the symbol level cannot exploit the time-varying CSI of the different subcarriers or of the different relays. More advanced techniques such as adaptive subcarrier allocation are required [10], [11].

Moreover, because relay-assisted OFDM transmissions are subjected to two-hop fading channels, dynamically rearranging the subcarriers at multiple relays may offer some additional diversity gains by appropriately pairing the subcarriers of the two hops. This subcarrier pairing philosophy was employed in [12], for both amplify-andforward (AF) and decodeand- forward (DF) relay-assisted single-user (SU) OFDM systems. This approach may also be applied in MU scenarios, such as in OFDMA, where the subcarriers of the two hops may be paired on a per-user basis, hence minimizing the multiuser interference (MUI). In addition, the corresponding resource allocation and MU scheduling may be carried out on a subbandgroup basis per user in the frequency domain (FD).

As a further advance, multiple relay selection schemes were investigated in an SU scenario when the CSI is available to the receivers but not to the transmitters. In this case, selecting multiple relays can increase the achievable cooperative/spatial diversity gain, but as a result, the potential selection diversity gain will be decreased when the total number of ORs is the same. Meanwhile, the interference among the multiple activated relays should be mitigated, which imposes further complexity on the transceivers. By contrast, when the CSI is available to both the receivers and the transmitters, optimum transmit beamforming was implemented through multiple relays. In that case, the signals from the relays coherently add at the receivers. Naturally, the elimination of the interference would require sophisticated algorithms, hence increasing the complexity imposed.

Therefore, carefully assigning the subbands/subcarriers in the context of OR can improve the diversity gain by avoiding the extra multirelay interference while retaining the MUI-free nature of SC-FDMA and OFDMA systems. Although both the dynamic relay selection (DRS and dynamic subband/subcarrier allocation (DSA) techniques can provide a power gain, conventional DSA-aided OR may not exploit both. The main reason is that the two hops of the relay channel limit the capacity to that of the lower capacity link of the two hops. In the context of OR-assisted SC-FDMA systems, both the AF and DF relays limit the attainable MU performance, depending on the quality of the first hop. The consideration of the received signal at multiple relays can further improve the performance of dynamic resource allocation (DRA)-aided OR systems. 
As a further advantage of channel-coded OR, the length of the interleavers combined with forward error correction (FEC) may be shortened, because the effects of fading are further randomized by OR. As a benefit, the delay of the entire FECcoded DF-relaying aided system is reduced. To the best of our knowledge, these issues have not been considered in the open literature.

In this paper, we propose two DRA strategies that were designed for the OR-assisted SC-FDMA uplink, where the ORs may invoke the soft-decision aid DF protocol. We assume that the multiple relays can exchange the pilot-aided channel-quality information (CQI) of all the users, facilitating the cooperation at the relays to carry out joint dynamic resource allocation (JDRA).1 Moreover, our assumption is that multiple relays participate in the multiway relaying (MWR) procedure for CQI exchange, as shown in Fig. 1(b), rather than for data exchange. The main contributions of this paper can be summarized as follows.

- In contrast to the aforementioned solutions, our focus is mainly on the energy efficiency of the DRA-aided OR-assisted SC-FDMA system, where the energy efficiency is quantified in terms of the energy consumption gain (ECG) and the energy reduction gain (ERG). In addition, we evaluated the impact of the channel estimation overhead and the MWR-aided CQI exchange overhead on the energy efficiency.

- We dedicate special attention to the "first-hop quality awareness" of our DRA schemes. In particular, we explore any potential extra selection diversity to reduce the transmit power required and/or to improve the attainable system performance. To this end, we combine DRS and DSA based on the associated first-hop transmission qualities. Meanwhile, two types of first-hop quality aware (FHQA) JDRA algorithms are investigated, depending on whether the source-to-relay $(\mathrm{S}-\mathrm{R})$ or the relay-to- destination (R-D) channel quality dominates the attainable performance when the system invokes either single or multiple antennas at the base station $(\mathrm{BS})$ receiver.

- Meanwhile, upon the "buffering delay awareness" of our interleaver-aided channel-coded systems, we quantify the benefits of OR combined with DSA in the context of interleaver-aided DF relaying for transmission over correlated fading channels in terms of reducing the interleaving delay and/or the total transmit power through joint OR and DSA.

- Our investigations involve the turbo equalization [26] in the FD, i.e., the minimum-mean-square-error (MMSE)- criterion-based frequency-domain linear equalizer (FD-LE) using iterative joint equalization and decodin. In addition, the performance benefits of both single-antenna and multiantenna-aided BS receivers have been considered for designing the bit-interleaved coded modulation (BICM) aided SCFDMA system.

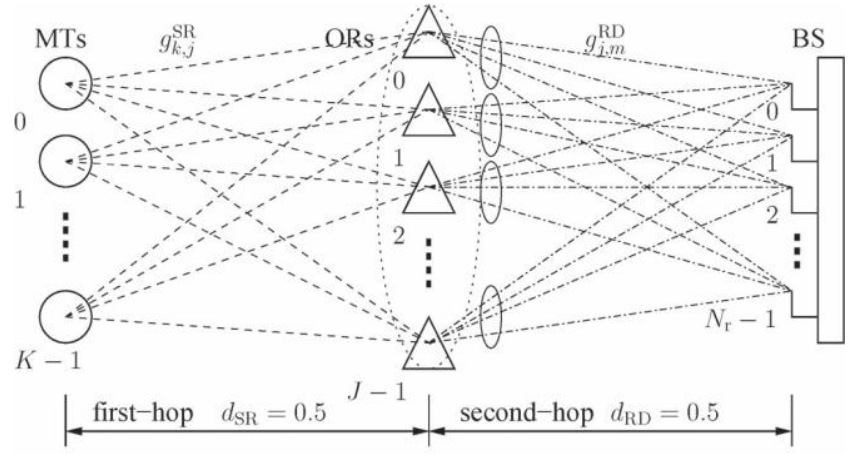

(a)

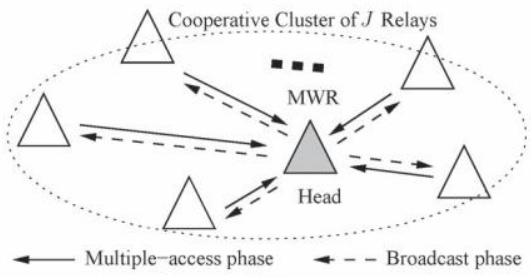

(b)

Figure 1: OR-based MU SC-FDMA uplink through CQI exchange. (a) Opportunistically relayed SC-FDMA uplink. (b) Pilot-aided CQI exchange in a cooperative relay cluster by assuming MWR.

This project is organized as follows. Section II presents about the Dynamic Resource Allocation systems. In Section III, we analyze the combination of BICM and SC-FDMA. Performance evaluation and comparisons are given in Section IV. Section V presents the conclusion.

\section{DRA FOR OR}

In the OR channels that we considered, both the spatial- and spectral-domain resources offered by multiple relays may be explored for power reduction. To achieve a selection diversity gain, the DRS allows each user to benefit from exploring $\mathrm{J}$ different S-R channels and J R-D channels. The corresponding complex-valued fading envelope may be deemed to be independent and identically distributed (i.i.d.) for each of these links of the resulting virtual MIMO scheme. Furthermore, the DSA beneficially rearranges the MU's signals for transmission over the most appropriate subband groups for the second-hop relaying. In addition, experiencing frequency-selective fading in the DRS-assisted OR scheme may provide an additional MU diversity gain for the system. Therefore, the beneficial combination of DRS and DSA can assign appropriate desired subband groups for conveying the MUs' signals regenerated at the appropriate relays, each of which may dynamically serve MUs.

FHQA JDRA: Although the conventional combination of DRS and DSA achieves a diversity gain through beneficial subband allocation and relay selection, the degree of freedom that is associated with beneficially allocating MU's signals across the entire set of $(\mathrm{M} \times \mathrm{J})$ subband groups of the $\mathrm{J}$ relays has not been fully exploited. Unless near-error-free decoding at the DF relays is possible, the MU's signals that were received and forwarded by the DF relays may result in error propagation at the BS due to the first-hop transmissions in terms of the S-R CQI. This phenomenon motivates us to design and investigate the first-hop quality-aware (FHQA) JDRA schemes conceived for OR-assisted SC-FDMA. We assume that the CSIs of the $\mathrm{K} \mathrm{S}-\mathrm{R}$ links and the $(\mathrm{M} \times \mathrm{J} \times \mathrm{Nr})$ $\mathrm{R}-\mathrm{D}$ links are perfectly estimated at each relay's receiver. We 
also assume that these cooperating relays can exchange their CQIs. More specifically, as mentioned in Section I, the ( $\mathrm{J}-1)$ relays in Fig. 1(b) [24] transmit their CQI to an appropriately selected relay that acts as a cluster head, which then broadcasts the CQI back to the $(\mathrm{J}-1)$ relays. The relevant decisions are made at the relay rather than at the BS. This implies that the BS's receiver does not require the CQI of the first hop.

In this section, the following three schemes are investigated:

1) conventional combination of DRS and DSA, denoted as DRS-DSA;

\section{2) FHQA JDRA approach-1, denoted as JDRA-1; and}

3) the FHQA JDRA approach-2, denoted as JDRA-2. The DRS-DSA scheme does not require any cooperation among the localized relays; hence, it is controlled by the BS, depending on the R-D CQI. By contrast, both the FHQA JDRA schemes rely on cooperation among the localized relays; hence, it is controlled by the relay cluster head. The flowcharts of FHQA JDRA schemes are portrayed in Fig. 2.

\subsection{CQI}

There are several reasons for grouping the $\mathrm{N}$ subbands of each user together for relaying. The first reason is related to the relevant complexity consideration, because searching for the optimal solution on a group-by-group rather than a subbandbysubband basis is significantly less complex. Another reason for grouping the $\mathrm{N}$ subbands of each user together is because each user's TD symbol generated after first-hop channel equalization at the relay is related to all the $\mathrm{N}$ elements in $\mathrm{yR}, \mathrm{f} \mathrm{kj}$ due to the well-understood nature of the IDFT employed at the SC-FDMA receiver. Therefore, we use the average $\mathrm{S}-\mathrm{R}$ channel attenuation of the $\mathrm{N}$ subbands of the kth user's signal received by the jth relay for quantifying the corresponding channel quality, which may be expressed as

$$
g_{k, j}^{\mathrm{SR}}=\frac{1}{N} \sum_{n=0}^{N-1}\left|h_{k, j, n}^{\mathrm{SR}, \mathrm{f}}\right|^{2}
$$

We also assume that the $\mathrm{BS}$ invokes $\mathrm{Nr}$ receiver antennas, where the sum of channel attenuation averaged over multiple antennas may be considered as a relevant quality metric for the overall channel effects. The average attenuation of the $m$ th group of $\mathrm{N}$ subbands in the channel spanning from the jth relay to the BS is given by

$$
g_{j, m}^{\mathrm{RD}}=\frac{1}{N} \sum_{u=m N}^{m N+N-1} \sum_{n_{\mathrm{r}}=0}^{N_{\mathrm{r}}-1}\left|h_{j, u, n_{\mathrm{r}}}^{\mathrm{RD}, \mathrm{f}}\right|^{2}
$$

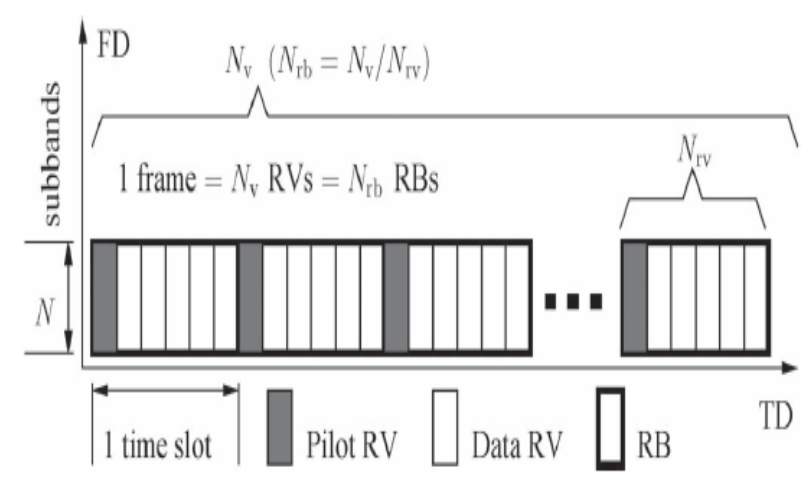

Figure 2: Resource allocation frame structure in the SCFDMA uplink scheme in Fig. 1(a).

\subsubsection{Relaying Frame Structure}

Observe in Fig. 2 that, in our DRA-aided OR system, each SC-FDMA symbol in the FD is referred to as a resource vector (RV), where the components of the RV are the original symbols that modulate each of the subbands. Furthermore, Nrv consecutive RVs in the TD constitute a resource block (RB) in the uplink during a single time slot, which constitutes the basic unit of resource allocation. The first RV may be regarded as the reference, i.e., the pilot RV in Fig. 4, which is known to both the transmitter and the receiver and, hence, may be used for channel estimation. Then, both the subband allocation and the relay selection valid for the corresponding time slot are particularly optimized for the channel quality that was estimated through this pilot RV. Hence, we have a total of $\mathrm{Nrb}=\mathrm{Nv} / \mathrm{Nrv} \mathrm{RBs}$ within a frame of Ns QAM symbols. In particular, the number of RVs Nrv per RB determines the transmission overhead required, including the synchronization sequences, the pilot RVs invoked for the estimation of the S-R and R-D links, and the duration of the CQI exchange in Fig. 1(b) for the FHQA JDRA schemes.4 Naturally, increasing the overhead provides more accurate channel estimation in support of the CQI exchange; hence, it may result in increased energy efficiency, although it may reduce the system's throughput. The Nrb RBs of each user are allocated by conventional static localized subband mapping during the first-hop transmissions, which reduces the effective throughput of the source MTs. However, during the second hop, each RB or, possibly, several consecutive RBs of a specific user may be rearranged at the appropriate relays, depending on the specific resource allocation schemes employed. Moreover, in the channel-coded system, the signal is interleaved and decoded on a frame-by-frame basis. Hence, an interleaver-depthdependent buffering delay is introduced when using iterative decoding at the DF relay. In addition, the specific number of consecutive RBs scheduled for concomitant transmission is typically determined by the normalized Doppler frequency fnd, which is usually carried out without changing the subband group and relay.

\section{BICM-SC-FDMA}

\subsection{Interleaving Algorithm}

The interleaving in IEEE 802.22 standard rearranges the position of its input samples based on a Turbo-Like algorithm. This algorithm uses the interleaving unit, $\mathrm{I}(\mathrm{k})$, iteratively so that the samples positions are updated after each iteration. The jth iteration for the algorithm is as follows: 


$$
\left\{\begin{array}{l}
I_{p, q}^{(0)}(k)=k \\
I_{p, q}^{(j)}(k) \mid=\left[K-p+k+q \cdot p \cdot\left[-k-p \cdot I_{p, q}^{(j-1)}(k)\right]_{K}\right]_{K}
\end{array}\right.
$$

Where $\mathrm{K}$, is the interleaving block size, $\mathrm{k}$ denotes the location of input samples, $\mathrm{j}$ presents the iteration number, and $\mathrm{p}$ and $\mathrm{q}$ are integer parameters. After the jth iteration, the positions of the samples at the productivity of the interleaver are obtained by Ip,q (j)(k). The operation $\mathrm{K}, \mathrm{X}$ modulo- $\mathrm{K}$, is expressed as follows:

$$
[X]_{K}=X-\text { floor }\left(\frac{X}{K}\right) \cdot K
$$

In IEEE 802.22 standard, both programmed bits and subcarriers are interleaved based on the variation in (1). The chosen parameters in the IEEE 802.22 standard for the bit interleaver are shown in TABLE I.

Table 1: Bit Interleaving Parameters.

\begin{tabular}{|c|c|c|c|c|c|c|c|}
\hline \multirow{2}{*}{ Block Size } & \multicolumn{3}{|c|}{$\begin{array}{c}\text { Interleaving } \\
\text { Parameters }\end{array}$} & Block Size & \multicolumn{3}{c|}{$\begin{array}{c}\text { Interleaving } \\
\text { Parameters }\end{array}$} \\
\hline K (bits) & $\mathrm{p}$ & $\mathrm{q}$ & $\mathrm{j}$ & $\mathrm{K}$ (bits) & $\mathrm{p}$ & $\mathrm{q}$ & $\mathrm{j}$ \\
\hline 48 & 16 & 2 & 2 & 960 & 6 & 2 & 3 \\
\hline 96 & 3 & 2 & 3 & 1008 & 36 & 2 & 1 \\
\hline 144 & 6 & 2 & 3 & 1056 & 16 & 2 & 3 \\
\hline 192 & 3 & 2 & 3 & 1152 & 36 & 2 & 1 \\
\hline 240 & 6 & 2 & 3 & 1248 & 3 & 2 & 2 \\
\hline 288 & 3 & 2 & 3 & 1344 & 6 & 2 & 3 \\
\hline 336 & 16 & 2 & 3 & 1440 & 40 & 2 & 2 \\
\hline 384 & 6 & 2 & 3 & 1536 & 6 & 2 & 3 \\
\hline 432 & 18 & 2 & 1 & 1632 & 3 & 2 & 3 \\
\hline 480 & 16 & 2 & 3 & 1680 & 40 & 2 & 2 \\
\hline 528 & 6 & 2 & 3 & 1728 & 36 & 2 & 1 \\
\hline 576 & 36 & 2 & 1 & 1824 & 48 & 2 & 1 \\
\hline 672 & 3 & 2 & 2 & 1920 & 48 & 2 & 1 \\
\hline 720 & 12 & 2 & 1 & 2016 & 16 & 2 & 3 \\
\hline 768 & 3 & 2 & 3 & 2112 & 16 & 2 & 3 \\
\hline 836 & 22 & 2 & 2 & 2208 & 3 & 2 & 3 \\
\hline 864 & 48 & 2 & 1 & 2304 & 16 & 2 & 3 \\
\hline
\end{tabular}

\subsection{System Level Simulation}

In order to hardware implementation of the interleaver, we have to shorten the interleaver expression into the arithmetic and logic operations. Also, a set of system level simulations is necessary to determine the Word Lengths (WLs) of the variables. Next, the suitable hardware is assigned to every operation.

To determine the WLs of the variables all the states of the interleaver are explored by MATLAB and the minimum number of bits for every variable is obtained which are exposed in TABLE II.

Table 2: Variables Word Length.

\begin{tabular}{|c|c|c|c|}
\hline Variable & $\begin{array}{c}\text { WL } \\
\text { (bits) }\end{array}$ & Variable & $\begin{array}{c}\text { WL } \\
\text { (bits) }\end{array}$ \\
\hline $\mathrm{X}_{\text {in }}$ & 18 & $1 / \mathrm{K}$ & 21 \\
\hline $\mathrm{X}_{\text {module_in }}$ & 12 & $\mathrm{X}_{1}$ & 33 \\
\hline $\mathrm{X}_{\text {out }}$ & 18 & $\mathrm{X}_{2}$ & 7 \\
\hline $\mathrm{I}$ & 12 & $\mathrm{X}_{3}$ & 12 \\
\hline
\end{tabular}

\subsection{Interleaver Architecture}

Generally, an interleaver receives input samples and then changes the position of the samples according to its transformation rule. A general architecture for the interleaver consists of three key modules. There are two RAM blocks for storing input samples and one address generator block to construct the position. When input data is written in a RAM, the output data is read as of the other one based on the generated address. The RAM blocks operations are exchanged behind every frame. Fig. 3 shows the ordinary architecture of the interleaver block.

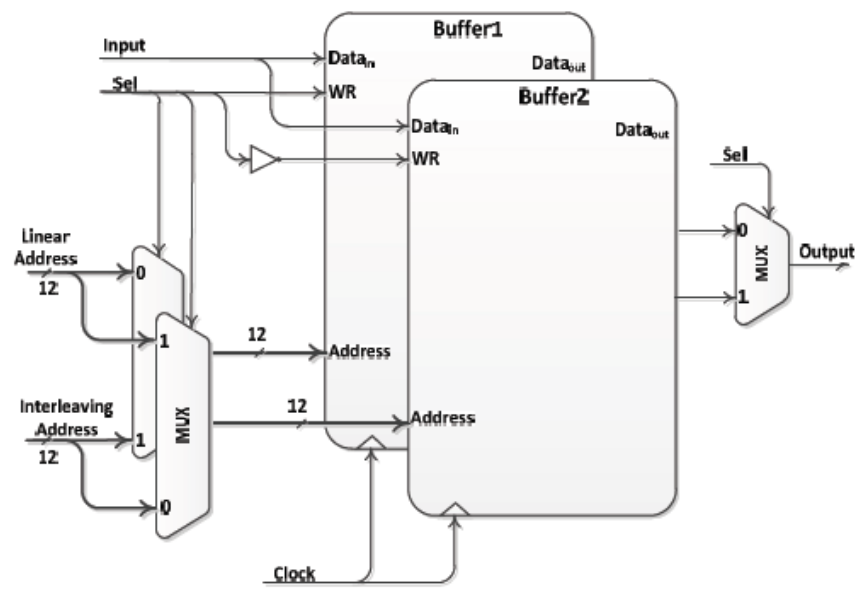

Figure. 3: General architecture of the interleaver.

The key execution challenge of the interleaver is its address generator plan while the implementation of other modules is simple.

Bit-interleaved coded modulation (BICM) is a flexible modulation/coding scheme which allows the designer to select a modulation constellation separately of the coding rate. This is since the output of the channel encoder plus the input to the modulator is divided by a bit-level interleaver. In order to increase spectral efficiency, BICM be able to join with high-order modulation schemes such as quadrature amplitude modulation (QAM) or phase shift keying. BICM is mainly well suitable for fading channels, and it simply introduces a small penalty in terms of channel quantity while compared to the coded modulation volume for together additive white Gaussian noise (AWGN) and fading channels. Furthermore, if the so-called BICM with iterative decoding (BICM-ID) is used, the demapper and decoder iteratively swap information, improving the system performance. On the receiver's side of BICM, the dependability metrics are designed for the coded bits under the shape of logarithmic likelihood ratios, or simply L-values. These metrics are later deinterleaved and further used by the soft-input channel decoder. This thesis deals with the probabilistic categorization of the L-values designed by the demapper while BICM is used in combination with high order QAM schemes. Three assistances are included in this thesis. In Paper A the issue of the probabilistic modeling of the extrinsic L-values for BICM-ID is addressed. First with a easy piece-wise linear model of the L-values obtained via the max-log estimate, expressions for the possibility density functions (PDFs) for Gray-mapped 16-QAM are originate. The developed logical expressions are then used to proficiently calculate the so-called extrinsic information transfer functions of the demapper, and they are as well compared with the histograms of the L-values obtained during 
numerical simulations. In Paper B closed-form expressions for the PDFs of the L-values in BICM by Gray mapped QAM constellations are developed. Based on these expressions, two simple Gaussian mixture approximations that are logically tractskill are also projected. The developments are used to powerfully calculate the BICM channel volume and to develop bounds on the coded bit-error rate while a convolutional code is used.

The coded performance of a hybrid automatic replicate request based on constellation rescheduling is also evaluated. In Paper $\mathrm{C}$ closed-form expressions for the PDFs of the $\mathrm{L}$ values in BICM transmissions with Gray-mapped QAM constellations over fully-interleaved fading channels are derived. The outcome are extraordinary for a Rayleigh fading channel, though, developments for the general case of a Nakagami-\$m\$ case are also integrated. By means of the developed expressions, the performance of BICM transmissions using convolutional and turbo codes is efficiently evaluated. The BICM channel volume for dissimilar fading channels and constellation sizes is also calculated.



Figure 4: Block diagram of BICM

\subsection{Convolution Encoder}

A convolution encoder encodes data prior to transmission over a channel. The encoded data is to be received on the other end of the channel and decoded via the Viterbi decoder. The convolution encoder computes and adds redundant bits to the data to be transmitted for more reliable transfer in an error-prone channel. This encoder is commonly used in wireless, cellular and satellite communications applications.

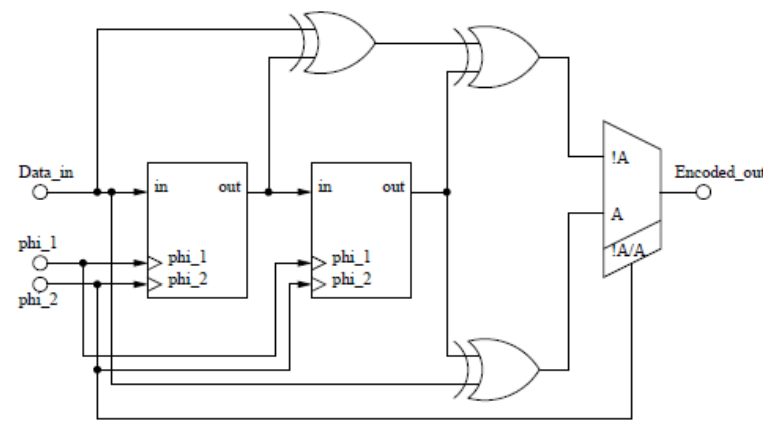

Figure 5: Block Diagram of a 3-bit Convolution Encoder

A block diagram of the convolution encoder can be seen in Figure 5.

The Trellis diagram of a convolution encoder dictates its output and next state. The Viterbi decoder on the other end of the channel follows the trellis diagram backwards to decode the data and correct any errors. The Trellis diagram for our 3bit encoder can be seen in Figure 3. The Trellis diagram is interpreted such that a state transition always occurs from right to left, and once the transition is made, you begin again on the left side of the diagram, in the appropriate current state.

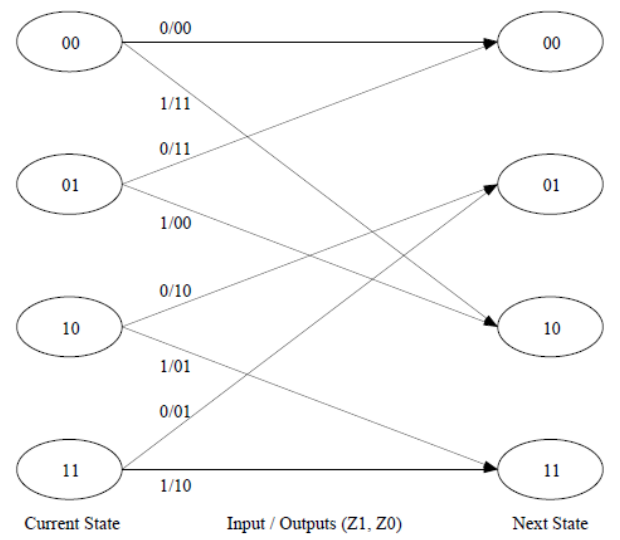

Figure 6: Trellis Diagram of a 3-bit Convolution Encoder

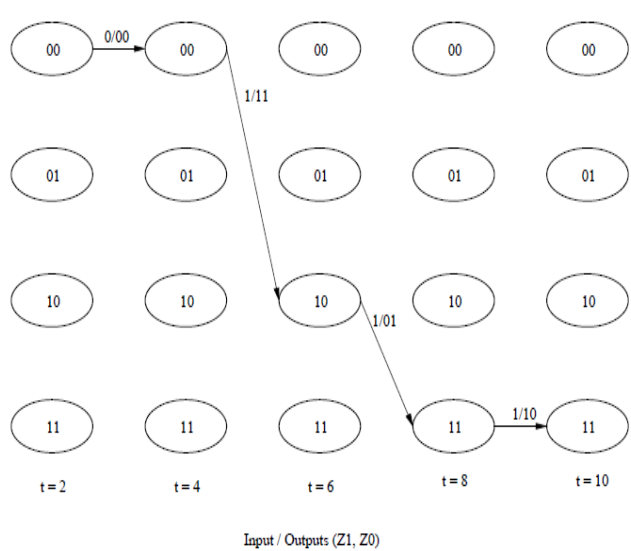

Figure 7: Trellis Diagram of a Step Input

\section{SIMULATION RESULTS}

In this section, the performance achieved by the various resource allocation schemes that rely on DF-aided OR is characterized in the context of channel-coded SC-FDMA systems upon varying the fading channel conditions.



Figure 8: Comparison of OR assisted SC-FDMA and BICM-SC-FDMA 
Fig. 6 depicts the BER versus Eb/N0 performance comparison of soft-DF-based OR systems for interleaver lengths of $\mathrm{Nc}=$ 720 and 7200 coded bits per frame, respectively. In particular, the performance of the single relay- aided system for $\mathbf{J}=1$ degrades when a fading channel suffers from increased TD correlation associated with a reduced normalized Doppler frequency fnd. This scenario may result in a series of burst errors within a coded bit stream associated with length of Nc over long-term deep fading, where the interleaver with limited depth is no longer effective.

\section{CONCLUSION}

In this paper, we have proposed and investigated two novel FHQA JDRA schemes to improve the reliability and energy efficiency of the soft-DF-based OR-assisted SC-FDMA uplink. By exploiting the first-hop quality for the joint design of DRS and DSA at the relays, the proposed FHQA JDRA algorithms outperform the conventional DRS-DSA and achieve up to 2-dB power reduction in channel-coded systems. When using a SC-FDMA DT benchmark, both FHQA JDRA schemes attain an ERG of $91 \%$ upon invoking a single-antenna BS and an ERG of up to $7.4 \%$ offered by the JDRA-2 scheme in the multiantenna-aided scenario, whereas their counterparts consume significantly more power. Furthermore, to decrease the buffering delay and reduce the transmit power, the interleaver depth of the proposed coded OR systems may be shortened by increasing the number of relays and invoking the soft-DF protocol when communicating over highly correlated fading channels.

\section{ACKNOWLEDGEMENTS}

This research was partially supported by the SEARCHiN project (FP6-042467, Marie Curie Actions). We would like to also thank Ala Hawash, Andreas Manoli, Anton Deik, Bilal Farraj, and Constantinos Savvides for their support and help in the implementation of MashQL.

\section{REFERENCES}

[1] K. J. R. Liu, A. K. Sadek, W. Su, and A. Kwasinski, Cooperative Communications and Networking. Cambridge, U.K.: Cambridge Univ. Press, 2008.

[2] A. Bletsas, A. Khisti, D. P. Reed, and A. Lippman, "A simple cooperative diversity method based on network path selection," IEEE J. Sel. Areas Commun., vol. 24, no. 3, pp. 659-672, Mar. 2006.
[3] H. G. Myung and D. J. Goodman, Single Carrier FDMA: A New Air Interface for Long Term Evolution. Hoboken, NJ: Wiley, 2008.

[4] F. Khan, LTE for 4G Mobile Broadband: Air Interface Technologies and Performance. Cambridge, U.K.: Cambridge Univ. Press, 2009.

[5] C. Y. Wong, R. S. Cheng, K. B. Letaief, and R. D. Murch, "Multiuser OFDM with adaptive subcarrier, bit and power allocation," IEEE J. Sel. Areas Commun., vol. 17, no. 10, pp. 1747-1758, Oct. 1999.

[6] T. Keller and L. Hanzo, "Adaptive multicarrier modulation: A convenient framework for time-frequency processing in wireless communications," Proc. IEEE, vol. 88, no. 5, pp. 611-640, May 2000.

[7] K. B. Letaief and Y. J. Zhang, "Dynamic multiuser resource allocation and adaptation for wireless systems," IEEE Wireless Commun., vol. 13, no. 4, pp. 38-47, Aug. 2006.

[8] T. Liu, C. Yang, and L.-L. Yang, "A low-complexity subcarrier-power allocation scheme for frequencydivision multiple-access systems," IEEE Trans. Wireless Commun., vol. 9, no. 5, pp. 1564-1570, May 2010.

[9] O. Nwamadi, X. Zhu, and A. K. Nandi, "Dynamic physical resource block allocation algorithms for uplink Long Term Evolution," IET Commun., vol. 5, no. 7, pp. 1020-1027, May 2011.

[10] Z. Han, T. Himsoon,W. P. Siriwongpairat, and K. J. R. Liu, "Resource allocation for multiuser cooperative OFDM networks:Who helps whom and how to cooperate," IEEE Trans. Veh. Technol., vol. 58, no. 5, pp. 2378-2391, Jun. 2009.

[11] O. Duval, Z. Hasan, E. Hossain, F. Gagnon, and V. Bhargava, "Subcarrier selection and power allocation for amplify-and-forward relaying over OFDM links," IEEE Trans. Wireless Commun., vol. 9, no. 4, pp. 1293-1297, Apr. 2010.

[12] W. Dang, M. Tao, H. Mu, and J. Huang, "Subcarrierpair-based resource allocation for cooperative multirelay OFDM systems," IEEE Trans. Wireless Commun., vol. 9, no. 5, pp. 1640-1649, May 2010. 